\title{
Overt is no better than covert when rehearsing visuo-spatial information in working memory
}

\author{
Richard Godijn • Jan Theeuwes
}

Published online: 20 July 2011

(C) The Author(s) 2011. This article is published with open access at Springerlink.com

\begin{abstract}
In the present study, we examined whether eye movements facilitate retention of visuo-spatial information in working memory. In two experiments, participants memorised the sequence of the spatial locations of six digits across a retention interval. In some conditions, participants were free to move their eyes during the retention interval, but in others they either were required to remain fixated or were instructed to move their eyes exclusively to a selection of the memorised locations. Memory performance was no better when participants were free to move their eyes during the memory interval than when they fixated a single location. Furthermore, the results demonstrated a primacy effect in the eye movement behaviour that corresponded with the memory performance. We conclude that overt eye movements do not provide a benefit over covert attention for rehearsing visuo-spatial information in working memory.
\end{abstract}

Keywords Attention - Eye movements · Working memory

Several lines of research have shown that spatial selection mechanisms are involved in maintaining visuo-spatial information in working memory (e.g., Awh, Anllo-Vento, \& Hillyard 2000; Awh, Jonides, \& Reuter-Lorenz 1998; Awh et al., 1999; Lawrence, Myerson, \& Abrams 2004; Smyth \& Scholey, 1994; Tremblay, Saint-Aubin, \& Jalbert 2006). It has been suggested that covert shifts of attention or overt movements of the eyes function as a rehearsal

R. Godijn $(\bowtie) \cdot J$. Theeuwes

Department of Cognitive Psychology, Vrije Universiteit,

Van Der Boechorststraat 1,

1081 BT Amsterdam, The Netherlands

e-mail: rj.godijn@psy.vu.nl mechanism within visuo-spatial working memory (e.g., Awh \& Jonides, 2001; Tremblay et al., 2006). In fact, Baddeley (1986) already suggested such a role for the oculomotor system, analogous to the articulatory rehearsal of verbal material. However, even without the execution of eye movements, observers can select the memorised locations through the allocation of covert attention. Indeed, it is yet unclear whether eye movements provide any benefit for visuo-spatial working memory relative to covert attention. Even though there is a strong relationship between covert attention and eye movements (e.g., Deubel \& Schneider, 1996; Hoffman \& Subramaniam, 1995; Kowler, Anderson, Dosher, \& Blaser 1995; Rizzolatti, Riggio, \& Sheliga 1987), in some circumstances these mechanisms are dissociated (Belopolsky \& Theeuwes, 2009), and therefore it is feasible that they have different effects on working memory.

The view that covert attention is a rehearsal mechanism that allows spatial information to be maintained in working memory gained support from a study by Smyth and Scholey (1994). In their study, participants performed a Corsi block task, which required them to remember the order in which the stimuli had been presented. It was found that secondary tasks requiring attention shifts performed during the retention interval impaired recall in this task (see also Smyth, 1996). This indicated that covert attention to the stimulus location was required during the retention interval to keep that location in working memory. Further evidence for a role of covert attention was provided by Awh, Jonides, and Reuter-Lorenz (1998), who found enhanced visual processing at locations that were held in working memory, suggesting that covert attention was directed at these to-be-remembered locations. Also, neural imaging and ERP studies of working memory tasks have demonstrated neural activity patterns 
(Awh et al., 1999, 2000; Jha, 2002) that were similar to the effects of covert attention.

In contrast to the covert attentional rehearsal view, Tremblay et al. (2006) have argued that rehearsal of visuo-spatial information is achieved by eye movements. This oculomotor rehearsal hypothesis is consistent with several lines of research that have pointed to some sort of relationship between memory and eye movements. Several studies have shown that participants regularly look at the previous location of a cued object, even if this location has become empty (e.g., Altmann, 2004; Richardson \& Spivey, 2000; see also Ferreira, Apel, \& Henderson 2008). It has also been shown that participants execute eye movements while forming a mental image and that these eye movements are related to the contents of the image being formed (e.g., Brandt \& Stark, 1997; Johansson, Holsanova, \& Holmqvist 2006; Laeng \& Teodorescu, 2002). Laeng and Teodorescu further demonstrated that the eye movement pattern during imagery was correlated with the eye movement pattern during the encoding of the image, as well as that the similarity in these eye movement patterns predicted performance. Furthermore, studies by Lawrence et al. (2004) and Pearson and Sahraie (2003) showed that secondary tasks involving attention or eye movements interfere with spatial working memory, and that eye movements interfere to a greater extent than covert shifts of attention. This implies that the effect of eye movements on working memory goes beyond the accompanying shift of attention.

The only study to date that has directly examined whether the oculomotor system plays a role in rehearsal for visuo-spatial information in working memory is Tremblay et al. (2006). In their study, participants were required to memorise the order of seven sequentially presented dots. After the presentation of the dots, there was a 10-s retention interval during which all seven dots were visible. Participants were required to indicate the correct order by pointing to the dots in their presented order. The results showed that during the retention interval, participants regularly moved their eyes to pairs of dots in their correct order. Moreover, recall performance increased as a function of the number of overtly rehearsed pairs of dots. In a second experiment, participants were prevented from using eye movements to rehearse the order, because they were required to alternate in fixating the two dots closest to the upper left corner. It was found that this secondary task impaired the recall of the dot order. Tremblay et al. interpreted these results as evidence that eye movements allow visuo-spatial information in working memory to be rehearsed and that this overt rehearsal contributes to working memory over and beyond covert rehearsal by attention.

However, there are a number of reasons to question this oculomotor rehearsal hypothesis. First, even in Tremblay et al.'s (2006) study, participants overtly rehearsed only a single pair of dots on about a third of the trials, and on another third of the trials did not even overtly rehearse a single pair of dots. The degree of overt eye movement rehearsal is therefore quite limited. Second, the correlational relationship between overt rehearsal and recall could well be due to the fact that both overt rehearsal and recall depend on how well the dot order was initially encoded and remembered at the start of the retention interval; if participants start the retention interval already with a poor memory of the order, they will not be able to adequately rehearse this order during the retention interval. Finally, it is important to note that for Tremblay et al., the actual stimuli were available in the external environment during the retention interval. This is in contrast to the studies of Awh and colleagues (e.g., Awh et al., 1998, 1999, 2000), in which participants memorised stimulus locations while the stimuli were no longer visible. Having the stimuli present allows participants to further encode the stimulus locations and is likely to motivate the execution of eye movements.

To examine whether overt eye movements contribute to the maintenance of visuo-spatial material in working memory, it is critical to directly manipulate eye movement behaviour. In the present study, we conducted two experiments to test the contribution of eye movements in visuo-spatial working memory. In both experiments, participants viewed displays containing a number of digits. After the digits were removed, there was a retention interval during which the display was blank. Either participants were instructed to move their eyes to a selection of the memorised locations (Exp. 1) or to keep their eyes fixed (Exp. 2) during the retention interval, or they were free to move their eyes (Exps. 1 and 2). After the retention interval, participants were required to indicate the locations of the digits in ascending numerical order with mouse responses. See Fig. 1 for an illustration of the display sequence. If eye movements facilitate the maintenance of visuo-spatial information in working memory, we expected that participants would respond more accurately to the subset of items that they fixated, and that memory performance would be better when they were free to move their eyes than when they were instructed to remain fixated on a single location.

\section{Experiment 1}

In Experiment 1, participants were instructed to make eye movements during the retention interval either to the memorised locations of the first three digits or to the memorised locations of the last three digits. Performance in 


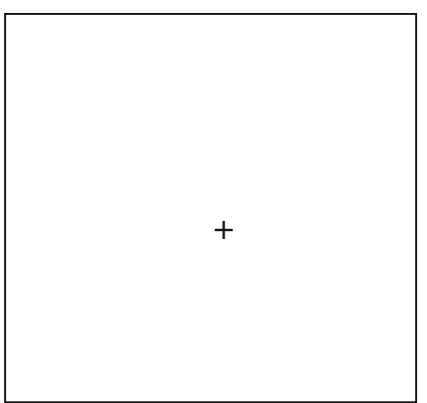

Fixation Display

(until key or mouse press)

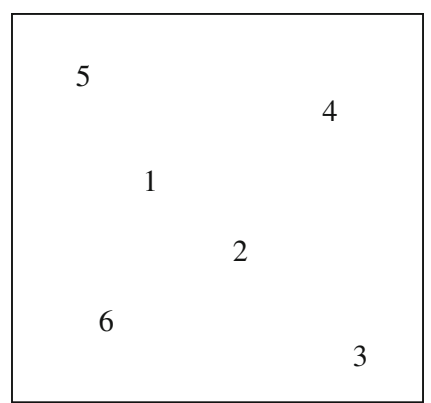

Digit Display

(10 sec.)

Fig. 1 The display sequence in Experiment 1. After pressing a key or mouse button, the digits 1-6 appeared on the display ("Digit Display"), and participants were required to remember their locations

these two conditions was compared to a condition with a free-eye-movement instruction, in which they were free to move their eyes however they wished. All to-beremembered stimuli were presented simultaneously, since this allowed us to examine oculomotor behaviour during encoding without the limitations imposed by a sequential presentation. Nevertheless, the required responses were in a predetermined order (from low to high numerical order), so that participants could not perform the task by memorising the global pattern of the digits. The predetermined serial order was expected to motivate the use of a rehearsal strategy (see, e.g., Tremblay et al., 2006).

\section{Method}

Participants After giving their informed consent, 9 students from Vrije Universiteit Amsterdam with normal or corrected-to-normal vision participated for course credit.

Apparatus A Pentium-based computer with a 21-in. colour monitor controlled the timing of the events and generated stimuli. Eye movements were recorded by means of an Eyelink 1000 tracker (SR Research), a video-based eyetracker sampling at $1000 \mathrm{~Hz}$. Each participant was tested in a dimly lit room. They held their head on a chinrest, located $70 \mathrm{~cm}$ away from the monitor.

Stimuli, procedure, and design At the start of each trial, participants viewed a small, white, centrally presented fixation cross on a black background. After they had fixated the central cross, a spacebar response recalibrated the gaze point to the central location. The fixation cross was then removed, and the digits 1-6 were presented in white at random locations within a window of $18.75^{\circ} \times 18.75^{\circ}$ of visual angle, with the restriction that each digit was at least $5^{\circ}$ from all other digits. This digit display was presented for

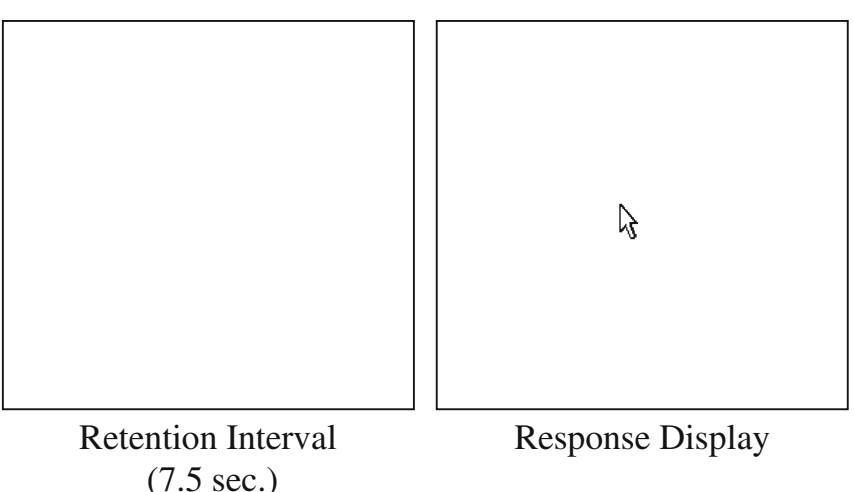

across a retention interval $(7.5 \mathrm{~s})$. Responses were given with nonspeeded mouse responses to the digits in ascending order

$10 \mathrm{~s}$, after which participants viewed a blank display for $7.5 \mathrm{~s}$ (the retention interval). After the retention interval, the mouse pointer appeared and the same digits were presented auditorily in ascending numerical order to probe memory of the digit locations. Participants were required to indicate the location of each digit by directing the mouse pointer to its memorised location and pressing the left mouse button. Each digit was presented directly after the participant had responded to the previous digit. See Fig. 1 for an illustration of the trial sequence. The mouse responses were nonspeeded, and participants were instructed to respond as accurately as they could. The eye movement instruction was varied between blocks. In some blocks, participants were free to move their eyes during the retention interval (the free condition). In other blocks, they were instructed to restrict their eye movements to the remembered locations of the first three digits (the first-three condition). In yet other blocks, they were instructed to move their eyes to the remembered locations of the last three objects (the last-three condition). In all blocks, participants were free to move their eyes before and after the retention interval. There were six blocks of 18 trials apiece (two blocks per eye movement instruction condition), and block order was counterbalanced across participants.

\section{Results}

Mouse response errors The proportion of order errors, in which participants indicated the correct location of a digit but in the wrong order, was very low (typically around 5\%), and therefore we decided to use the mean mouse response error as the dependent measure. For each mouse response, we calculated the deviation of the response from the digit location, and these values were averaged for each condition, 
resulting in a measure of mean mouse response error. Figure 2 shows the mean mouse response errors as a function of each condition. An ANOVA on mean mouse response error with the factors eye movement instructions (free, first-three, and last-three) and digit (1-6) revealed significant main effects of eye movement instructions, $F(2,16)=22.72, p<.001$, and digit, $F(5$, $40)=21.56, p<.001$, as well as a significant interaction between eye movement instructions and digit, $F(10,80)=$ 3.82, $p<.001$. Planned $t$ tests revealed significantly reduced mouse response errors, relative to the free eye movement condition, in the first-three condition for digit $1, t(8)=2.51, p<.04$, and digit $3, t(8)=2.40, p<.05$, as well as nonsignificant trends towards reduced errors for digit $2, t(8)=1.62, p<.15$, and digit $4, t(8)=1.86, p<$ .10. There were significantly increased mouse response errors, relative to the free eye movement condition, in the last-three condition for digit $2, t(8)=4.31, p<.01$, digit 3 , $t(8)=4.52, p<.01$, digit $5, t(8)=2.38$, and digit $6, t(8)=$ $4.73, p<.001$. Finally, there were nonsignificant trends towards increased errors in the last-three condition for digit $1, t(8)=1.63, p<.015$, and in the first-three condition for digit $6, t(8)=1.69, p<.15$.

Eye movements during digit display The fixation time on each digit was defined as the total amount of time participants fixated near a particular digit within a margin of $2.5^{\circ}$ (i.e., half the minimum distance between any two digits). The mean fixation times during the digit display are plotted in Fig. 3. An ANOVA on the mean fixation durations during the digit display revealed a significant main effect of digit, $F(5,40)=14.09, p<.001$, as well as a significant interaction between eye movement instructions and digit, $F(10,80)=5.66, p<.001$, but no significant effect of the eye movement instructions, $F(2$, 16) $=2.27, p>.10$. As can be seen in Fig. 3, participants

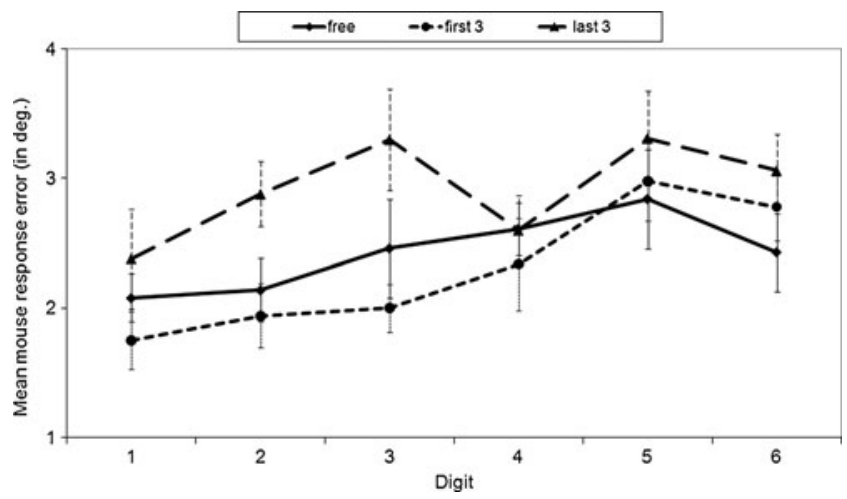

Fig. 2 Mean mouse response errors for each of the digits as a function of the eye movement instructions (free, first three, or last three) in Experiment 1

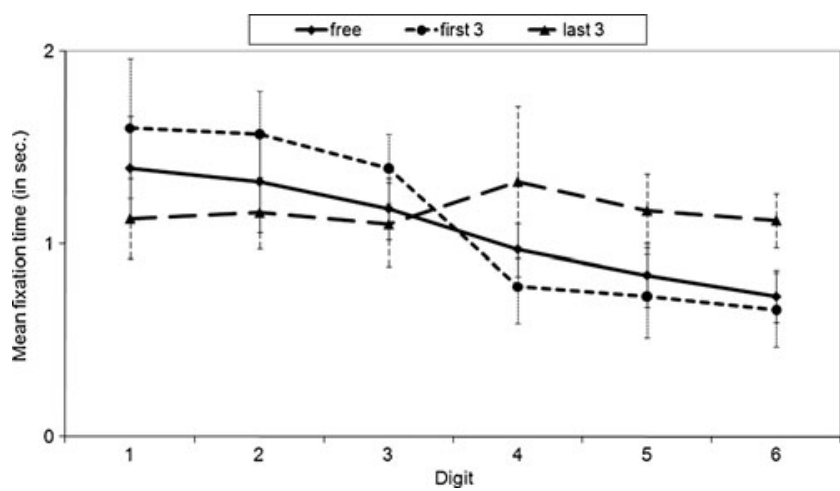

Fig. 3 Mean fixation times near each of the digit locations during the digit display as a function of the eye movement instructions (free, first three, or last three) in Experiment 1

spent more time fixating digits that they would be required to fixate during the retention interval.

Eye movements during the retention interval The mean fixation times during the retention interval plotted in Fig. 4 reveal that participants followed the instructions in the first-three and last-three conditions to restrict their fixations to a subset of the remembered digit locations. In the free eye movement condition, a significant effect of digit was found, $F(1,5)=4.24, p<.01$, reflecting a primacy effect in eye movement behaviour. When the analyses were restricted to the to-be-fixated locations, the effect of digit failed to reach significance for the first-three condition, $F(2,16)=2.01, p>.15$, as well as for the lastthree condition, $F(2,16)=3.40, p>.05$.

\section{Discussion}

Experiment 1 showed that response accuracy was significantly affected by which locations were chosen as saccade goals. Executing saccades to the memorised locations of the

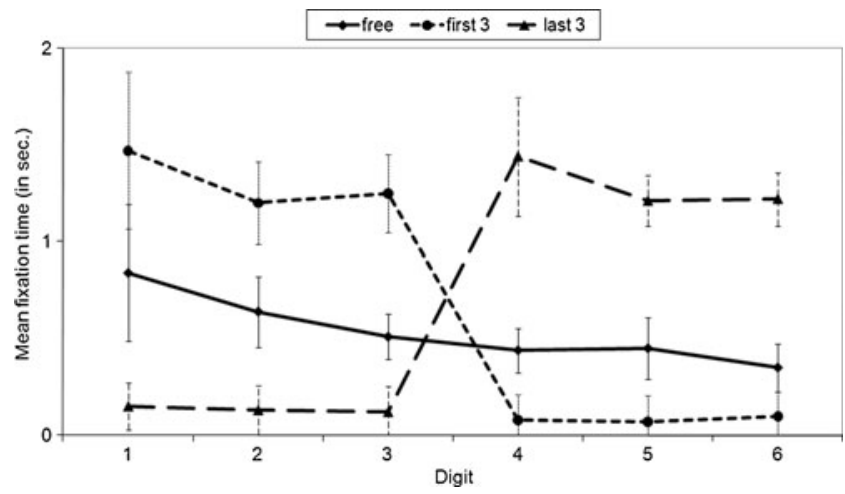

Fig. 4 Mean fixation times near each of the digit locations during the retention interval as a function of the eye movement instructions (free, first three, or last three) in Experiment 1 
first three digits provides a benefit for these locations. However, executing saccades to the memorised locations of the last three digits did not improve memory for these locations, and even impaired memory for digits 5 and 6 , as well as for the nonfixated digits. Executing saccades to the higher digits was clearly an inferior strategy, and it is therefore understandable that participants in the free eye movement condition spent more time fixating the lower digits. This implies that eye movements have a strong effect on working memory performance, but, given the impaired memory performance in the last-three condition, they do not necessarily improve performance.

\section{Experiment 2}

The results of Experiment 1 demonstrated that eye movements do not necessarily improve performance in a visuo-spatial working memory task. However, in the free eye movement condition, participants regularly chose to execute saccades during the retention interval. It is still unclear whether these eye movements actually facilitated memory, since it is possible that covert attention can accomplish memory rehearsal just as well without the execution of eye movements. In order to further examine the role of eye movements in visuo-spatial memory rehearsal, in Experiment 2 we compared a free eye movement condition with a condition in which participants were instructed to remain fixated at a single location during the retention interval. Participants were allowed to select whichever fixation location they wished in the fixate condition, so that they were not forced to fixate a taskirrelevant location such as the central location. Thus, the only difference between the two eye movement instruction conditions was that in the fixate condition, participants fixated a single, freely chosen location, while in the free eye movement condition they were allowed to select as many freely chosen locations as they wished and were allowed to execute as many saccades as they wished.

If eye movements facilitate the maintenance of visuospatial information in working memory, we would expect that participants would respond more accurately when they were free to move their eyes than when they were instructed to remain fixated on a single location. In contrast, if eye movements do not contribute to visuo-spatial working memory to a greater extent than covert attention, we would expect recall accuracy to be no greater in the free eye movement condition than in the fixate condition.

In addition to changing the eye movement instructions relative to Experiment 1, we also decided to manipulate the eye movement instructions within blocks of trials, instead of the between-block manipulation of Experiment 1. The results of Experiment 1 demonstrated that participants already altered their eye movement strategies during encoding, depending on the eye movement instructions for the retention interval. Since there is evidence that eye movements during encoding can affect memory recall (Saint-Aubin, Tremblay, \& Jalbert 2007), we decided to provide the eye movement instructions after the removal of the digit display.

\section{Method}

Participants After giving their informed consent, 14 students from Vrije Universiteit Amsterdam with normal or corrected-to-normal vision participated for course credit.

Stimuli, procedure, and design The experiment was the same as Experiment 1, except for the eye movement instructions, which were varied within blocks. On each trial, $200 \mathrm{~ms}$ after the removal of the digits, a small white fixation point was presented at the centre of the screen. After $1,000 \mathrm{~ms}$, the colour of the fixation point changed to either red or green for $1,000 \mathrm{~ms}$, after which it was removed from the screen. If the fixation point was red, participants were required to fixate a single location during the retention interval (fixate condition). If the fixation point was green, participants were free to move their eyes any way they wished (free condition). Participants were informed that they were free to pick any location on the screen to fixate in the fixate condition. This instruction meant that participants were not forced to fixate a task-irrelevant location (i.e., the central location), so the only difference between the free and fixate conditions was whether participants could execute saccades during the retention interval. There were five blocks of 18 trials, and each block had 9 trials from the fixate condition and 9 from the free condition, and the order of trials was randomised within blocks.

\section{Results}

Mouse response errors Fig. 5 shows the mean mouse response errors for each digit in the fixate condition and the free condition. An ANOVA on the mean mouse response errors with the factors eye movement instructions (free, fixate) and digit (1-6) revealed a significant main effect of digit, $F(5,65)=10.10, p<.001$, and a significant interaction between eye movement instructions and digit, $F$ $(5,65)=2.54, p<.04$, but no effect of eye movement instructions, $F(1,13)=1.19, p>.25$. As can be seen in Fig. 5, the interaction reflects a slight advantage for the fixate condition for the lowest digits, which turns in to a slight advantage for the free condition for the higher digits. 


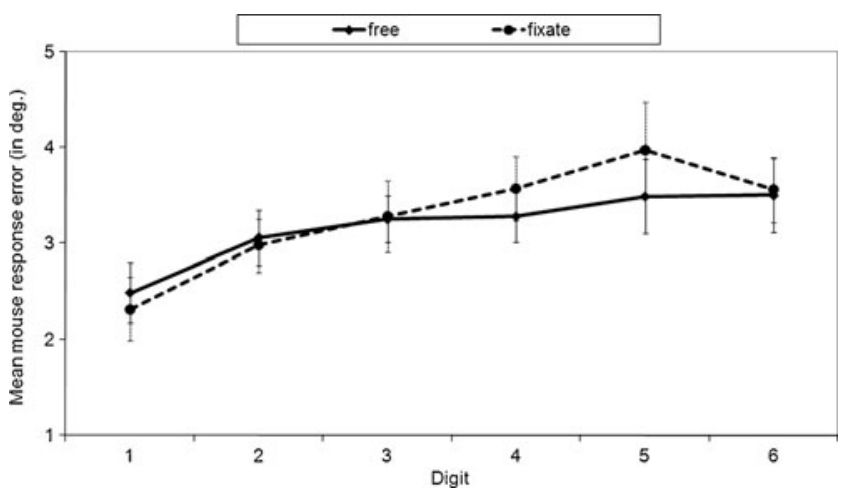

Fig. 5 Mean mouse response errors for each of the digits as a function of the eye movement instructions (free or fixate) in Experiment 2

However, only the planned $t$ test for the digit 5 reached significance, $t(13)=2.42, p<.04$, while there were nonsignificant trends for digit $1, t(13)=1.64, p>.10$, and digit $4, t(13)=1.65, p>.10$. After a Bonferroni correction for multiple comparisons, none of the $t$ tests came close to significance.

Eye movements during digit display The mean fixation times during the digit display are plotted in Fig. 6. An ANOVA on the mean fixation durations during the digit display revealed a significant main effect of digit, $F(5$, $65)=6.65, p<.001$, but no effect of eye movement instructions, $F(1,13)<1$, and no interaction between eye movement instructions and digit, $F(5,65)=2.11, p>.05$. Indeed, any nonsignificant differences between the free condition and the fixate condition must be due to chance, given the fact that participants were unaware of the eye movement instructions while they were encoding the digits.

Eye movements during retention interval The mean fixation times during the retention interval are plotted in Fig. 7. There was no effect of eye movement instruc-

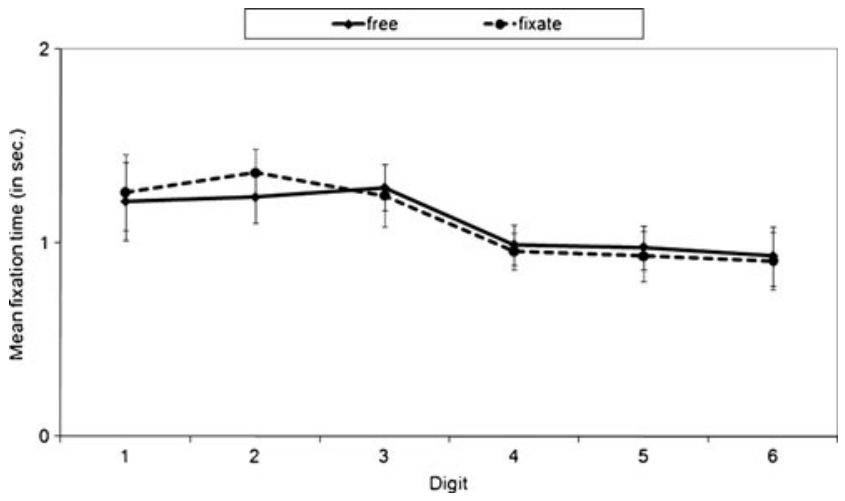

Fig. 6 Mean fixation times near each of the digit locations during the digit display as a function of the eye movement instructions (free or fixate) in Experiment 2

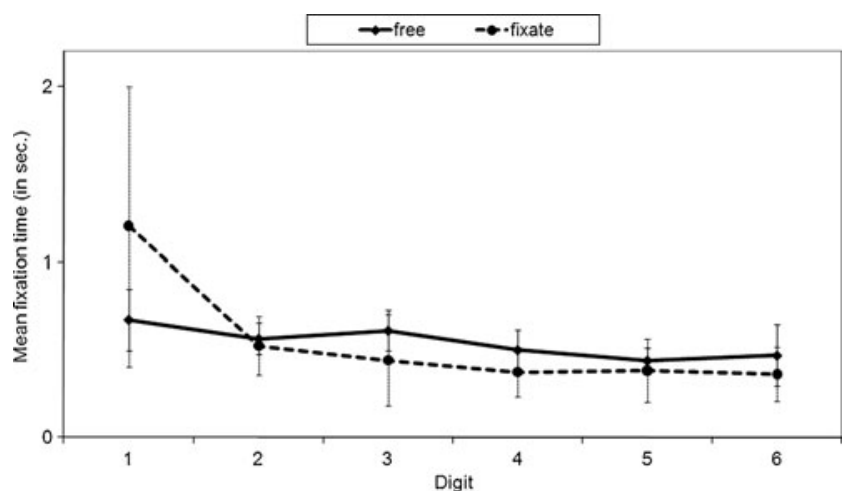

Fig. 7 Mean fixation times near each of the digit locations during the retention interval as a function of the eye movement instructions (free or fixate) in Experiment 2

tions, $F(1,13)<1$, but there was a significant effect of digit, $F(5,65)=3.33, p<.01$, and a significant interaction between eye movement instructions and digit, $F(5,65)=$ 2.63, $p<.04$. Planned comparisons for each digit between the fixate and free eye movement conditions did not produce any significant differences, although there were nonsignificant trends towards more time spent at digit 1 in the fixate condition, $t(13)=1.56, p>.10$, and more time spent at digits 3 and 4 in the free condition, $t(13)=1.57$, $p>.10$, and $t(13)=2.12, p>.05$. As can be seen in Fig. 7, the main difference between the two conditions, responsible for the significant interaction, is that some participants typically chose to fixate the remembered location of digit 1 in the fixate condition. However, different fixation strategies were chosen by different participants, and the range of mean fixation durations on digit 1 between participants varied from 0.4 to $5.3 \mathrm{~s}$, and because of this large variation between participants, none of the planned comparisons reached significance.

We also examined the total numbers of eye movements (greater than $2^{\circ}$ of visual angle) executed during the retention interval in the fixate and free conditions, to check whether participants followed the eye movement instructions. On average, participants executed 1.1 saccades in the fixate condition and 14.3 saccades in the free condition, thereby confirming that they chose a single location to fixate in the fixate condition, while they executed several saccades in the free eye movement condition.

\section{Discussion}

Experiment 2 has shown that executing sequences of saccades between the memorised locations of the digits did not improve memory recall relative to a condition in which participants were required to fixate a single location. Even though participants executed approximately 14 
saccades on average during the retention interval in the free eye movement condition, against just a single saccade in the fixate condition (towards a location that would be fixated throughout the retention interval), the mean mouse response errors did not differ significantly between the two conditions. Additionally, we replicated the finding of Experiment 1 that participants prioritised the lower digits; they fixated longer on the lower digits during encoding, and this corresponded with a better memory recall for the lower digits.

\section{General discussion}

The present study examined the effect of eye movements on the retention of visuo-spatial information in working memory. Participants performed a visuo-spatial working memory task in which they were required to memorise the locations of a number of digits in ascending numerical order. The critical manipulation was the eye movement instructions for the retention interval. Experiment 1 demonstrated that the instruction to move the eyes to the memorised locations of the first three digits resulted in a selective improvement for these locations, whereas the instruction to execute saccades to memorised locations of the last three digits resulted in an impairment, not only for the nonfixated digits, but even for some of the fixated digits (5 and 6). Experiment 2 demonstrated that the freedom to execute multiple saccades between the digits during the retention interval did not result in better memory recall relative to a condition in which participants fixated a single location.

The results of the present study indicate that overt eye movements do not provide any benefit relative to the covert attentional mechanisms that have already been shown to be involved in working memory (e.g., Awh et al., 1998, 1999, 2000; Awh \& Jonides, 2001; Jha, 2002; Smyth, 1996; Smyth \& Scholey, 1994). Nevertheless, participants in the free eye movement conditions of both experiments tended to fixate the lower digits more than the higher digits during the retention interval, and these were precisely the digits that were best memorised. This finding should not be mistaken as evidence for the superiority of overt rehearsal over covert rehearsal. In fact, this finding merely shows that participants prioritise the lower digits over the higher digits, as in the classic primacy effect, because this results in better overall memory performance. It is plausible that even when participants do not execute eye movements during the retention interval, either because of the task instructions or because of a deliberate strategy, they covertly attend more to the lower digits than to the higher digits. Moreover, the priority given to the lower digits can also be seen by the eye movement behaviour during the digit display; even during the encoding of the digits, more time is spent on the lower digits.

The memory recall impairment in the last-three condition and the priority given to the lower digits suggests that serial memory recall benefits when rehearsal prioritises the items with the lowest serial order position. Given the tight link between attention and eye movements (e.g., Deubel \& Schneider, 1996; Hoffman \& Subramaniam, 1995; Kowler et al., 1995), the instruction to saccade to the last three digits during the retention interval also hindered covert attentional deployment to the memorised locations of the first three digits. Despite the fact that eye movements during the retention interval did not improve performance, in the present study participants generally chose an eye movement strategy when they were free to do so. This is consistent with previous research that has shown that participants regularly execute saccades during visual imagery or working memory tasks. For example, Altmann (2004) presented visual scenes containing two people and two objects, and after removing the scene asked participants to listen to a related sentence. It was found that participants regularly moved their eyes to the now empty locations that corresponded to the spoken sentence. Similarly, Richardson and Spivey (2000) presented in their Experiment 1 a sequence of talking heads, each in a separate quadrant. Each of these heads delivered a certain fact to be remembered. Subsequently, a test fact was presented that was related to one of the four presented facts, and participants were required to indicate whether or not this test fact was true. After hearing the test fact, participants frequently executed saccades to the corresponding quadrant, even though there was now nothing to be seen there. Richardson and Spivey found that looking at the critical quadrant did not improve memory performance (see also Hoover \& Richardson, 2008, for a similar finding). They interpreted their findings as support for O'Regan's (1992) view of the world as an external memory store. According to this view, observers make use of the general stability of the external world by returning their eyes to a remembered location, since this will often provide better information than an internal memory search would. Altmann proposed that these eye movements to empty remembered locations are the result of an episodic trace, which includes a spatial index of the object or event. Activating the trace activates the spatial index, and this automatically triggers a saccade to its remembered location. In Richardson and Spivey's and Altmann's studies, these eye movements were not seen as actually improving memory, but Ferreira et al. (2008) proposed an alternative. Their model posits an integrated memory representation consisting of associated visual, linguistic, conceptual, and spatial-location information. Activation of any part of the representation facilitates the activation of the other parts. Thus, an eye movement to a remembered location will facilitate the 
retrieval of visual, linguistic, and conceptual information, because they are linked to the spatial-location information. In this view, the saccade to a remembered location is functional, not because the object might still be there in the external world, but because of internal memory facilitation. Note, however, that this memory facilitation would also be expected to work for covert shifts of attention. According to the reasoning behind this model, when an observer covertly shifts attention to a remembered location, this activation of spatial-location information would also be expected to facilitate retrieval of other parts of the memory trace.

Although previous studies that have examined looking at remembered locations have nicely demonstrated a strong link between working memory and spatial selection (covert or overt), selecting a single location in response to a memory cue is quite different from strategically rehearsing multiple spatial locations. It is clear that in the present task, eye movements did not facilitate overall memory performance. This does not imply, however, that eye movements never facilitate memory performance. In the present study, the display was kept completely blank, and there was no visual information on the display that could be used to improve memory. In contrast, in Tremblay et al. (2006) the stimuli were present during the retention interval, so that only the presentation order of the stimuli was uncertain. This makes overt rehearsal of the order much easier than in a blank display. In this manner, overt rehearsal becomes not just an implicit memory aid, but makes use of further visual processing to improve memory. However, when the display is completely blank, eye movements are directed to empty locations, and there is no external feedback concerning the accuracy of the eye movements. Yet even with a completely blank display, it is likely that participants are able to exploit some external visual information. Specifically, they might memorise spatial locations in relation to the frame of the monitor. Therefore, while the present study has provided evidence against the view of the oculomotor system as a rehearsal mechanism for visuo-spatial material, observers might under certain circumstances move their eyes to certain locations to benefit from external cues.

Another possible strategy that participants might adopt is to make use of some form of verbal encoding. Most likely, participants verbally rehearse the name of the digit when they are attending a specific digit. For example, if they attend (overtly or covertly) the locations of 1,2 , and 3 , in that order, they could bind the spatial locations with the names of the digits and repeat "one," "two," "three" in their phonological loop. Indeed, Guérard, Tremblay, and Saint-Aubin (2009) found that phonological similarity of letters impaired the recall of their spatial locations, suggesting that phonological rehearsal was involved in memorising the spatial locations of these letters. It might even be possible that participants verbally encode and rehearse the locations in a more elaborate way, by using words like "left," "right," "top," bottom," and "centre." For example, the digits from Fig. 1 might be recoded as "three, bottom right," "five, top left," and so forth. However, with six digits to be remembered, such elaborate verbal encoding would overtax the abilities of phonological rehearsal, which can typically rehearse just a few seconds of verbal material (see Baddeley, 2003, for a review). Nevertheless, in the present study, random locations on the display were chosen (with the only restrictions being that the digits were presented within a window of $18.75^{\circ} \times$ $8.75^{\circ}$ of visual angle, with each digit at least $5^{\circ}$ from all other digits) and no framework or grid was used (in contrast with, e.g., Laeng \& Teodorescu, 2002, and Brandt \& Stark, 1997), in order to prevent relatively easy recoding of the locations. Participants were required to be as precise as possible, and a verbal code like "top left" would generally not result in accurate responses. Viewed within the context of Baddeley's (1986, 2000; Baddeley \& Hitch, 1974) working memory model, it is likely that participants would make use of both the phonological loop - rehearsing the names of the digits - and the visuo-spatial sketchpadrehearsing (covertly and/or overtly) the spatial locations of these digits. However, even though the possibility of more elaborate verbal encoding cannot be entirely excluded, such encoding would have occurred equally in the free eye movement condition and the fixate condition of Experiment 2. During encoding of the digit display of that experiment, participants did not know whether they would be free to make multiple saccades during the retention interval, so the strategies during encoding could not have differed between these conditions.

The present findings are consistent with the view that covert attention rehearses visuospatial information in working memory. Although there was no direct measure of covert attention in the present study, previous research had demonstrated that observers do attend to locations when maintaining visuo-spatial information in working memory (e.g., Awh et al., 1999, 2000; Jha, 2002; see also Awh \& Jonides, 2001). Furthermore, allocating attention to a secondary task disrupts the maintenance of visuo-spatial information in working memory (Awh et al., 1998; Smyth \& Scholey, 1994, 1996), suggesting that attentional allocation is required. However, given the evidence for a role of covert attention and the present evidence that eye movements do not provide a further benefit, it remains to be explained why secondary tasks involving eye movements disrupt working memory performance to a greater extent than those that merely involve covert attention (Lawrence et al., 2004; Pearson \& Sahraie, 2003). It is important to realise that secondary tasks requiring eye movements also have implications for attentional selection. It is well known 
that attention and eye movements are strongly related (see, e.g., Corbetta et al., 1998; Rizzolatti et al., 1987; see also Awh et al., 2006) and that attention precedes the eyes to the saccade goal (e.g., Deubel \& Schneider, 1996; Hoffman \& Subramaniam, 1995; Kowler et al., 1995). It is quite plausible that the attention requirements of saccade goal selection are different from those of covertly attending without the intention of moving the eyes. In fact, recent evidence from single-cell recordings in monkeys has shown that covert shifts of attention during a visual search task can take a mere $40 \mathrm{~ms}$ (Buschman \& Miller, 2009), which is much faster than executing sequences of eye movements. If a task requires covert attention to lead the way for overt eye movements, it is likely that attention will operate more slowly and be less flexible than can be achieved during an extended period of fixation. In addition, secondary tasks requiring eye movements also result in the shifting of the frame of reference. If observers can hold on to spatial locations in working memory in retinal coordinates, then the execution of eye movements will require the memorised locations to be updated across eye movements.

The finding that eye movement execution did not improve overall memory performance in the present study does not preclude a more limited role for the oculomotor system in the rehearsal of visuo-spatial material in working memory. That is, the oculomotor-rehearsal hypothesis could be reconciled by suggesting that the actual execution of the eye movement is not what facilitates working memory of visuo-spatial material, but rather the programming of eye movements. From this perspective, rehearsal is achieved by an implicit eye movement - an eye movement program without actual motor acts - much as rehearsal of verbal material can be achieved by implicit speech, or articulatory rehearsal without actual motor acts (e.g., Baddeley, 1986; Baddeley, Thompson, \& Buchanan 1975; Buchsbaum \& D'Esposito, 2008).

Although moving the eyes between multiple digits did not improve memory recall in the present study, it also did not cause an impairment. This suggests that rehearsal during memory retention can operate equally well overtly, with sequences of eye movements, and covertly, with attention shifts during fixation.

Open Access This article is distributed under the terms of the Creative Commons Attribution Noncommercial License which permits any noncommercial use, distribution, and reproduction in any medium, provided the original author(s) and source are credited.

\section{References}

Altmann, G. T. M. (2004). Language-mediated eye movements in the absence of a visual world: The "blank screen paradigm. Cognition, 93, B79-B87. doi:10.1016/j.cognition.2004.02.005.
Awh, E. Armstrong, K. M., \& Moore, T. (2006). Visual and oculomotor selection: links, causes and implications for spatial attention. Trends in Cognitive Sciences, 10, 124-130.

Awh, E., \& Jonides, J. (2001). Overlapping mechanisms of attention and spatial working memory. Trends in Cognitive Sciences, 5, 119-126. doi:10.1016/S1364-6613(00)01593-X.

Awh, E., Jonides, J., \& Reuter-Lorenz, P. A. (1998). Rehearsal in spatial working memory. Journal of Experimental Psychology. Human Perception and Performance, 24, 780-790. doi:10.1037/ 0096-1523.24.3.780.

Awh, E., Jonides, J., Smith, E. E., Buxton, R. B., Frank, L. R., Love, T., et al. (1999). Rehearsal in spatial working memory: Evidence from neuroimaging. Psychological Science, 10, 433-437. doi:10.1111/1467-9280.00182.

Awh, E., Anllo-Vento, L., \& Hillyard, S. A. (2000). The role of spatial selective attention in working memory for locations: Evidence from event-related potentials. Journal of Cognitive Neuroscience, 12, 840-847. doi:10.1162/089892900562444.

Baddeley, A. D. (1986). Working memory. Oxford: Oxford University Press, Clarendon.

Baddeley, A. (2000). The episodic buffer: A new component of working memory? Trends in Cognitive Sciences, 4, 417-423. doi:10.1016/S1364-6613(00)01538-2.

Baddeley, A. (2003). Working memory: Looking back and looking forward. Nature Reviews Neuroscience, 4, 829-839. doi:10.1038/ nrn1201.

Baddeley, A. D., \& Hitch, G. (1974). Working memory. In G. H. Bower (Ed.), The psychology of learning and motivation: Advances in research and theory, vol 8 (pp. 47-89). New York: Academic.

Baddeley, A. D., Thomson, N., \& Buchanan, M. (1975). Word length and the structure of short-term memory. Journal of Verbal Learning and Verbal Behavior, 14, 575-589. doi:10.1016/ S0022-5371(75)80045-4.

Belopolsky, A. V., \& Theeuwes, J. (2009). When are attention and saccade preparation dissociated? Psychological Science, 20, 1340-1347. doi:10.1111/j.1467-9280.2009.02445.x.

Brandt, S. A., \& Stark, L. W. (1997). Spontaneous eye movements during visual imagery reflect the content of the visual scene. Journal of Cognitive Neuroscience, 9, 27-38. doi:10.1162/ jocn.1997.9.1.27.

Buchsbaum, B. R., \& D'Esposito, M. (2008). The search for the phonological store: From loop to convolution. Journal of Cognitive Neuroscience, 20, 762-778. doi:10.1162/jocn.2008.20501.

Buschman, T. J., \& Miller, E. K. (2009). Serial, covert shifts of attention during visual search are reflected by the frontal eye fields and correlated with population oscillations. Neuron, 63, 386-396.

Corbetta, M., Akbudak, E., Conturo, C. E., Snyder, A. Z., Ollinger, J. M., Drury, H. A., et al. (1998). A common network of functional areas for attention and eye movements. Neuron, 21, 761-773.

Deubel, H., \& Schneider, W. X. (1996). Saccade target selection and object recognition: Evidence for a common attentional mechanism. Vision Research, 36, 1827-1837. doi:10.1016/0042-6989 (95)00294-4.

Ferreira, F., Apel, J., \& Henderson, J. M. (2008). Taking a new look at looking at nothing. Trends in Cognitive Sciences, 12, 405-410. doi:10.1016/j.tics.2008.07.007.

Guérard, K., Tremblay, S., \& Saint-Aubin, J. (2009). The processing of spatial information in short-term memory: Insights from eye tracking the path length effect. Acta Psychologica, 132, 136-144. doi:10.1016/j.actpsy.2009.01.003.

Hoffman, J. E., \& Subramaniam, B. (1995). The role of visual attention in saccadic eye movements. Perception \& Psychophysics, 57, 787-795. doi:10.3758/BF03206794. 
Hoover, M. A., \& Richardson, D. C. (2008). When facts go down the rabbit hole: Contrasting features and objecthood as indexes to memory. Cognition, 108, 533-542. doi:10.1016/j.cognition. 2008.02.011.

Jha, A. P. (2002). Tracking the time-course of attentional involvement in spatial working memory: An event-related potential investigation. Cognitive Brain Research, 15, 61-69. doi:10.1016/S09266410(02)00216-1.

Johansson, R., Holsanova, J., \& Holmqvist, K. (2006). Pictures and spoken descriptions elicit similar eye movements during mental imagery, both in light and in complete darkness. Cognitive Science, 30, 1053-1079. doi:10.1207/ s15516709 $\operatorname{cog} 0000 \_86$.

Kowler, E., Anderson, E., Dosher, B., \& Blaser, E. (1995). The role of attention in the programming of saccades. Vision Research, 35, 1897-1916. doi:10.1016/0042-6989(94)00279-U.

Laeng, B., \& Teodorescu, D.-S. (2002). Eye scanpaths during visual imagery reenact those of perception of the same visual scene. Cognitive Science, 26, 207-231. doi:10.1016/S0364-0213(01) 00065-9.

Lawrence, B. M., Myerson, J., \& Abrams, R. A. (2004). Interference with spatial working memory: An eye movement is more than a shift of attention. Psychonomic Bulletin \& Review, 11, 488-494. doi:10.3758/BF03196600.

O'Regan, J. K. (1992). Solving the "real" mysteries of visual perception: The world as an outside memory. Canadian Journal of Psychology, 46, 461-488. doi:10.1037/h0084327.

Pearson, D. G., \& Sahraie, A. (2003). Oculomotor control and the maintenance of spatially and temporally distributed events in visuo-spatial working memory. Quarterly Journal of Experimental Psychology, 56A, 1089-1111.

Richardson, D. C., \& Spivey, M. J. (2000). Representation, space and Hollywood Squares: Looking at things that aren't there anymore. Cognition, 76, 269-295. doi:10.1016/S0010-0277 (00)00084-6.

Rizzolatti, G., Riggio, L., \& Sheliga, B. M. (1987). Space and selective attention. In C. Umiltà \& M. Moscovitch (Eds.), Attention and performance $X V$ : Conscious and nonconscious information processing (pp. 231-265). Cambridge: MIT Press, Bradford Books.

Saint-Aubin, J., Tremblay, S., \& Jalbert, A. (2007). Eye movements and serial memory for visual-spatial information: Does time spent fixating contribute to recall? Experimental Psychology, 54, 264-272. doi:10.1027/1618-3169.54.4.264.

Smyth, M. M. (1996). Interference with rehearsal in spatial working memory in the absence of eye movements. Quarterly Journal of Experimental Psychology, 49A, 940-949. doi:10.1080/ 027249896392379.

Smyth, M. M., \& Scholey, K. A. (1994). Interference in immediate spatial memory. Memory \& Cognition, 22, 1-13. doi:10.3758/ BF03202756.

Smyth, M. M., \& Scholey, K. A. (1996). Serial order in spatial immediate memory. Quarterly Journal of al Psychology, 49A, 159-177. doi:10.1080/027249896392847.

Tremblay, S., Saint-Aubin, J., \& Jalbert, A. (2006). Rehearsal in serial memory for visual-spatial information: Evidence from eye movements. Psychonomic Bulletin \& Review, 13, 452-457. doi:10.3758/BF03193869. 